\title{
Singularities Analysis of the Jacobian Matrix Modified in the Continuation Power Flow: Performance Evaluation
}

\author{
A. Bonini Neto and D. A. Alves
}

\begin{abstract}
In the literature, a study to analyze stability and voltage instability is related to the $P-V$ curve (power versus voltage magnitude) and the maximum loading point (MLP) (point on the curve that separates the stable operation of the unstable). The maximum loading point may be consequent to a saddle node bifurcation (SNB) related to transmission capacity limit in an electrical system where the Jacobian matrix is singular, or limit induced bifurcation (LIB), related the reactive power limit of the generator, where the matrix is not singular. In this sense, it is presented in this second part of the paper the trajectory of the values of the determinants of the modified Jacobian matrices $\left(\left|\mathbf{J}_{\mathrm{m}}\right|\right)$ of these methods. A graphical analysis is also shown for a better understanding. The methods studied are: local parameterization $\left(\lambda\right.$ ou $\left.V_{k}\right)$, global parameterization (total real power losses $(\mathrm{Pa})$ and the quadratic equation in $P a$ plane in function of loading factor. The results are obtained to the two real large systems of 638 and 787 buses corresponding to part of South-Southeast Brazilian systems and of a 904-bus Southwestern American system.
\end{abstract}

Keywords - Voltage stability, Maximum loading point, Bifurcation, Continuation power flow, Continuation method.

\section{INTRODUÇÃO}

$\mathrm{N}$ ESSA parte do trabalho a singularidade da matriz $\mathbf{J}$ é verificada através da inversão do sinal do determinante no ponto de máximo carregamento (PMC), ou seja, o determinante é nulo exatamente no PMC, podendo ser verificado em sistemas de pequeno porte por meio do gráfico dos valores numéricos do determinante em função do carregamento $\lambda$. A dimensão da matriz quadrada $\mathbf{J}$ é da ordem de $\left(2 n_{\mathrm{PQ}}+n_{\mathrm{PV}}\right)$, onde $\mathrm{n}_{\mathrm{PQ}}$ e $n_{\mathrm{PV}}$ correspondem ao número de barras $\mathrm{PQ}$ e $\mathrm{PV}$, respectivamente. Na primeira parte do trabalho [1] foi proposto um algoritmo por meio de um fluxograma para obtenção dos valores dos determinantes, o algoritmo baseia-se na divisão dos elementos da matriz jacobiana modifica $\left(\mathbf{J}_{\mathbf{m}}\right)$ por $n$ conforme a Fig. 1 a seguir.

Os métodos utilizados na primeira parte foram propostos em [2], [3] e [6 - 10]. Os resultados obtidos com estes métodos para os sistemas de grande porte mostram que as características de convergência dos métodos de fluxo de potência são melhoradas na região do PMC, possibilitando a sua determinação com a precisão desejada sem os problemas numéricos relacionados à singularidade da matriz $\mathbf{J}$.

A. Bonini Neto, Faculdade de Ciências e Engenharia - FCE, UNESP, Rua Domingos da Costa Lopes, 780, Bairro: Jd. Itaipu, 17602-496 - Tupã - Brasil (e-mail: bonini@tupa.unesp.br).

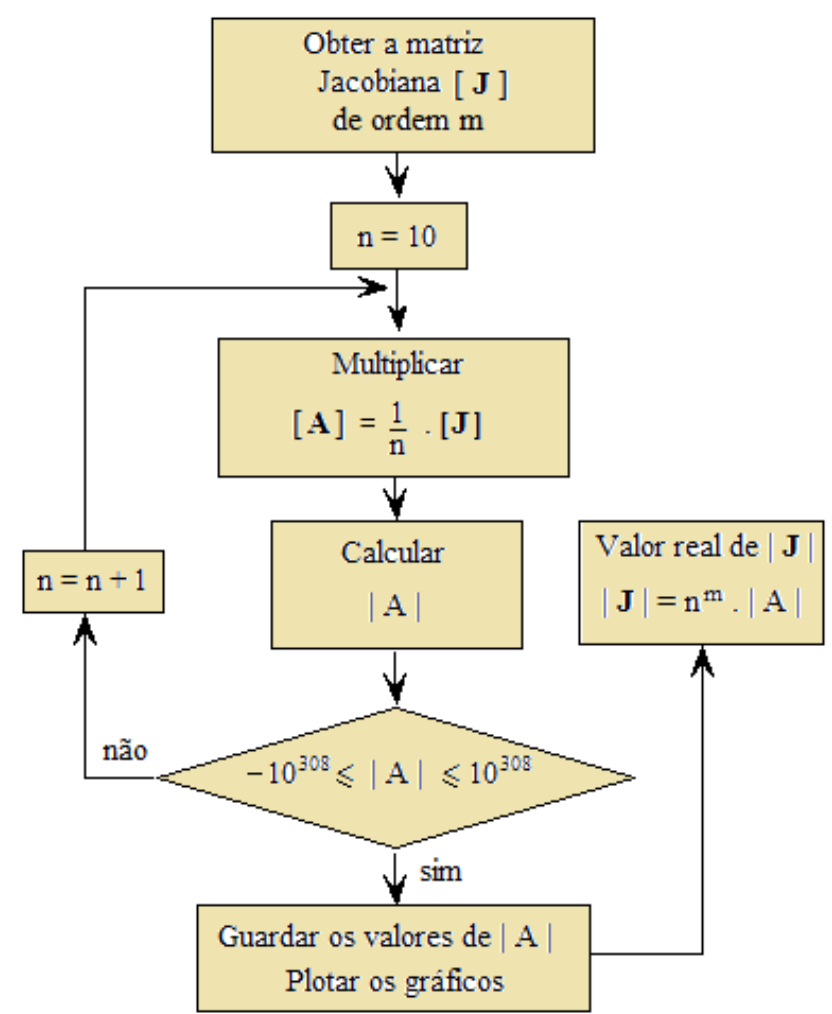

Figura 1. Fluxograma para obtenção dos valores dos determinantes de sistemas de grande porte.

Nesta parte também são apresentados os valores dos determinantes a fim de verificar as possíveis ocorrências de singularidades da matriz $\mathbf{J}_{\mathbf{m}}$ de cada método estudado, bem como o número de iterações necessárias para obtenção dos pontos representando a curva P-V. Outro aspecto importante mostrado neste trabalho são os valores dos determinantes de cada ponto pertencente à geometria da trajetória de soluções do problema de fluxo de carga continuado (FCC) parametrizado, explicitando que a impossibilidade de obtenção do PMC pelo FCC convencional se deve à problemas numéricos (singularidade). O mesmo já não ocorre para a matriz Jacobiana modificada $\mathbf{J}_{\mathbf{m}}$ quando da escolha adequada do parâmetro.

Todas as equações aqui citadas estão localizadas na primeira parte do trabalho proposto [1].

D. A. Alves, Departamento de Engenharia Elétrica, Faculdade de Engenharia de Ilha Solteira, UNESP, Av. Brasil, 56, 15385-000, Ilha Solteira Brasil (e-mail: dalves@dee.feis.unesp.br).

Corresponding author: A. Bonini Neto 


\section{RESUltados}

\section{Resultados para o Sistema Sul Sudeste Brasileiro de 638 barras}

Sistemas de grande porte como o Sul-Sudeste Brasileiro de 638 barras apresentam matrizes Jacobianas da ordem $1189 \times 1189$ para o FCC convencional e $1190 \times 1190$ para o FCC parametrizado, devido a inclusão de uma linha e uma coluna representando o novo parâmetro. Neste contexto, com o auxílio do algoritmo apresentado na Fig. 1 pode-se obter os valores dos determinantes de sistemas de grande porte.

A Fig. 2, 3(a) e 3(b) apresentam as curvas P-V da barra crítica $\left(\mathrm{V}_{150}\right)$ e perdas totais de potência ativa $(P a)$ versus fator de carregamento do sistema 638 barras, respectivamente. Os pontos " $a$ " e " $b$ " representam os pontos críticos da curva os quais serão analisados. A Fig. 4(a) mostra os valores dos determinantes em torno do ponto " $a$ " (PMC) para todos os métodos. Considerando o fluxo de carga convencional (FCC) (equação 4) ou $\lambda$ como parâmetro (equação 17) proposto por [2] para o traçado da curva $\mathrm{P}-\mathrm{V}$, pode-se observar que o valor do determinante neste caso é zero, acarretando em uma singularidade. Esses resultados também podem ser observados na tabela 1, quando há uma inversão no sinal do determinante.

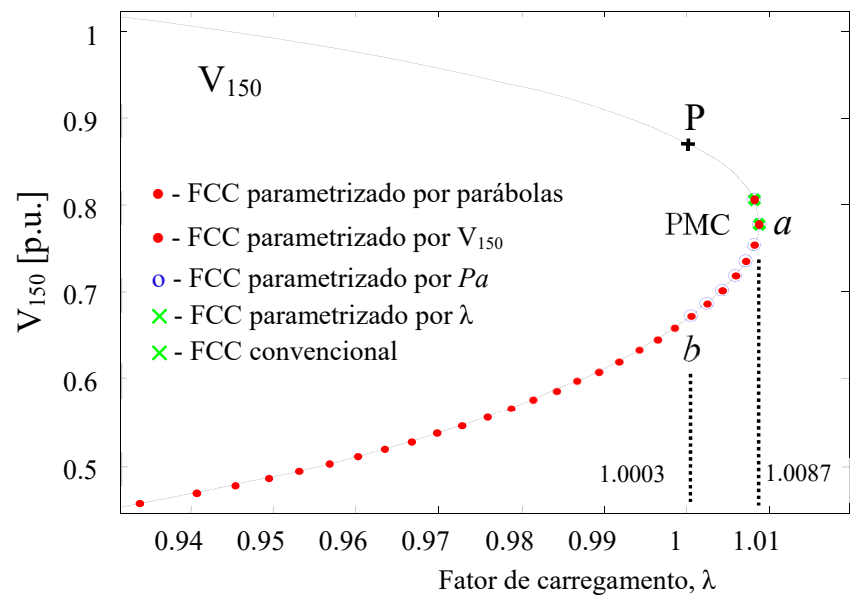

Figura 2. Comparação dos métodos no sistema 638 barras, resultados na curva P-V.

Já a Fig. 4(b), se o parâmetro for as perdas totais de potência ativa $\mathrm{Pa}$ (equação 30 ) da primeira parte do artigo e proposto por [3], a singularidade ocorre no ponto " $b "(1.0003,1.1077)$, ver Fig. 3(a), e que também corresponde ao ponto " $b$ " na curva P$\mathrm{V}$ da Fig. 2. Observa-se que este ponto se encontra na parte de baixo da curva, ou seja, após o ponto de máximo carregamento (PMC). A tabela 2 mostra os valores dos determinantes quando parametrizado por $P a$ em torno do ponto " $b$ ", pode-se notar que há uma mudança de sinal de $\left|\mathbf{J}_{\mathbf{m}}\right|$ quando o método passa por esse ponto. Os demais métodos (parametrizado por $\mathrm{V}_{150}$ (equação 18) proposto em [2] e parametrizado por parábolas (equação 36) proposto em [5]) não apresentaram singularidades em nenhum ponto da curva P-V quando a barra escolhida para o traçado for a barra crítica.
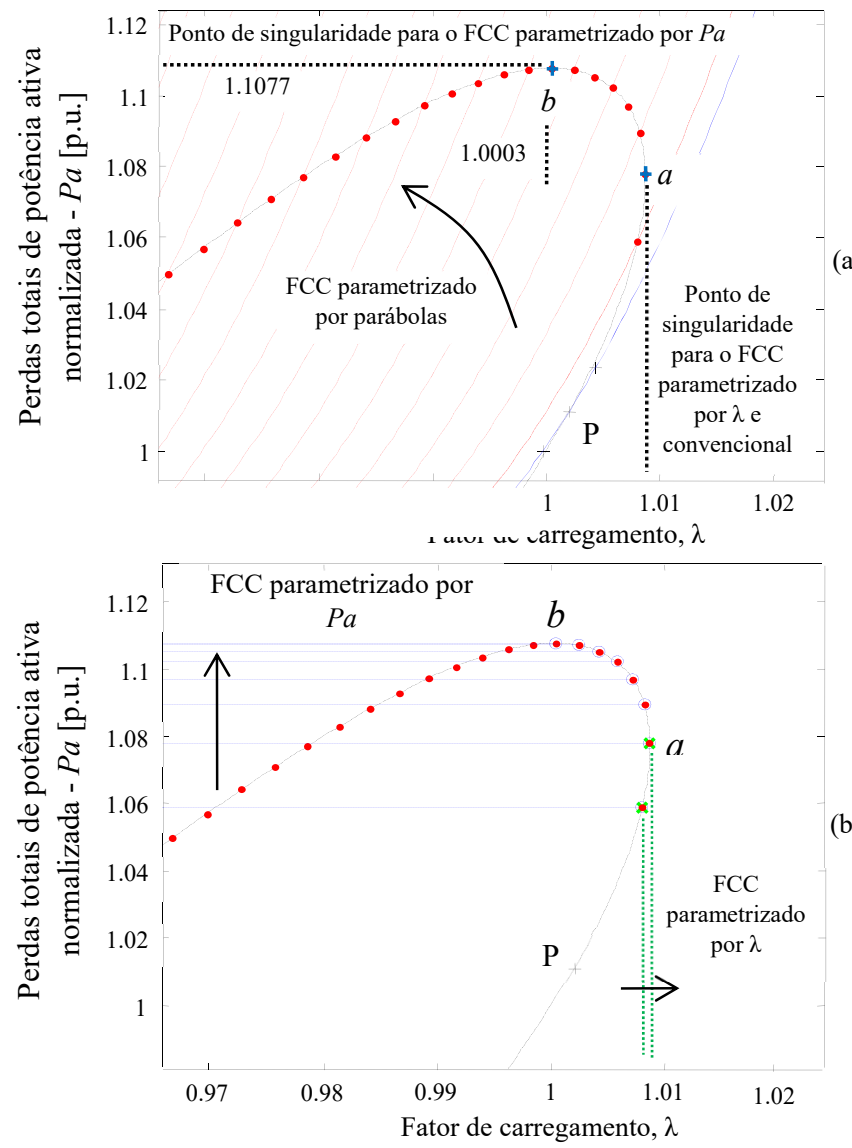

Figura 3. Comparação dos métodos no sistema 638 barras, resultados na curva $\lambda-P a$, (a) parametrização baseada em parábolas, (b) performance dos métodos parametrizado por $P a$ e $\lambda$.

Os valores de n no algoritmo proposto, para que seja possível a visualização e plotagem dos gráficos dos determinantes das matrizes $\mathbf{J}$ e $\mathbf{J}_{\mathbf{m}}$ do sistema Sul-Sudeste Brasileiro, foram entre 35 e 105 , ver tabela 6 . Para este sistema, o valor de $\mathrm{n}$ adotado foi de 58, com isso, os valores reais dos determinantes do FCC obtido por meio da equação:

$$
|\mathbf{J}|=\mathrm{n}^{\mathrm{m}} \cdot|\mathbf{A}|=58^{1189} \cdot|\mathbf{A}|
$$

são apresentados nas tabelas 1 e 2 , onde $|\mathbf{A}|$ é o valor do determinante obtido pelo MATLAB [4], que varia entre $-10^{308}$ e $10^{308}$ para cada método analisado e $\mathrm{m}$ é a ordem da matriz $\mathbf{J}$.

Em [5] foi proposto o uso da equação da parábola como parâmetro da continuação, para este trabalho, foi acrescentado o termo $P a_{c b}$ (valor de $P a$ no caso base), com o intuito de normalizar os dados, ficando:

$$
\Delta \mathrm{W}(\boldsymbol{\theta}, \mathbf{V}, \lambda, \alpha)=\frac{P a(\boldsymbol{\theta}, \mathbf{V})}{P a_{\mathrm{bc}}}-\left(\mathrm{a}(\lambda+\alpha)^{2}+\mathrm{b}(\lambda+\alpha)+\mathrm{c}\right)=0
$$

onde os coeficientes $\mathrm{a}, \mathrm{b}$ e $\mathrm{c}$ da função quadrática foram obtidos por meio da interpolação polinomial de Lagrange utilizando as seguintes fórmulas: 


$$
\begin{gathered}
\operatorname{Pn}(x)=\sum_{i=0}^{n} L_{i}(x) f\left(x_{i}\right) \\
L_{i}(x)=\prod_{j=0, j \neq i}^{n} \frac{\left(x-x_{j}\right)}{\left(x_{i}-x_{j}\right)}, i=0,1, \ldots, n
\end{gathered}
$$

A matriz jacobiana desse novo método passa a ser:

$$
\mathbf{J}_{\mathbf{m}}=\left[\begin{array}{ccc}
\mathbf{G}_{\boldsymbol{\theta}} & \mathbf{G}_{\mathbf{V}} & \mathbf{G}_{\lambda} \\
\partial P a / \partial \boldsymbol{\theta} & \partial P a / \partial \mathbf{V} & -(2 a(\lambda+\alpha)+b)
\end{array}\right]
$$

que é não singular nos pontos críticos " $a$ " e " $b$ " conforme visto nas Figs. 2, 3, 4 e 5.

Fig. 5(a) e (b) exibem comportamentos de $\lambda$ e $P a$ como uma função do parâmetro $\alpha$ (equação 5 ). O ponto " $a$ " corresponde ao PMC onde $\lambda$ atinge seu valor máximo, no caso de usar esta variável $\lambda$ como parâmetro, a matriz jacobiana modificada apresentará uma singularidade (tabela 1 ). $\mathrm{O}$ mesmo ocorre no ponto " $b$ ", usando o $P a$ como parâmetro, a matriz jacobiana modificada apresentará uma singularidade, neste caso logo após o PMC. No entanto, o PMC e toda a curva P-V podem ser facilmente calculados com o método parametrizado por parábolas porque a singularidade de $\mathbf{J}$ é removida quando $\alpha$ é utilizado como um parâmetro.
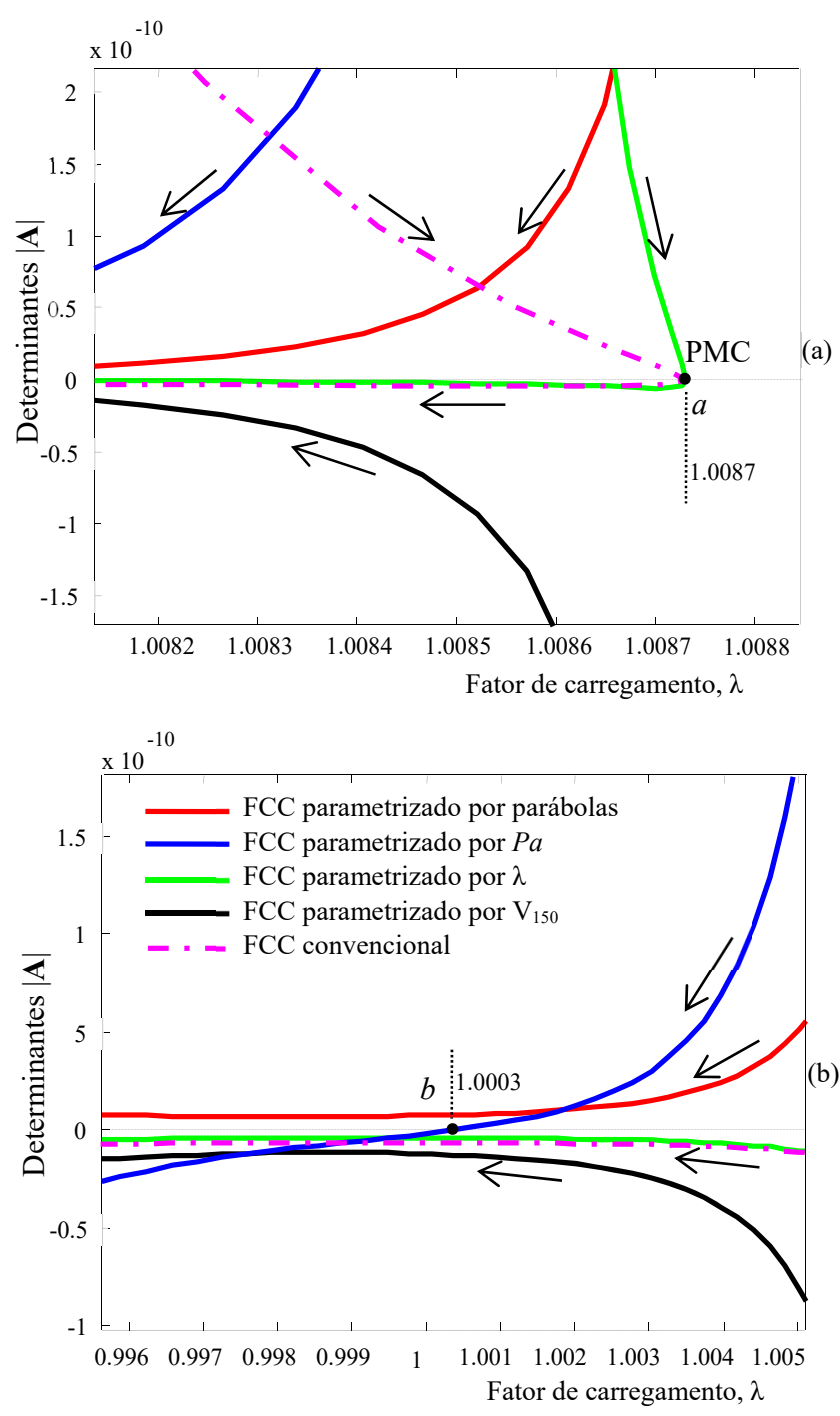

Figura 4. Determinantes dos métodos para o sistema 638 barras: (a) performance dos métodos no ponto " $a$ ", (b) performance dos métodos no ponto " $b$ ".

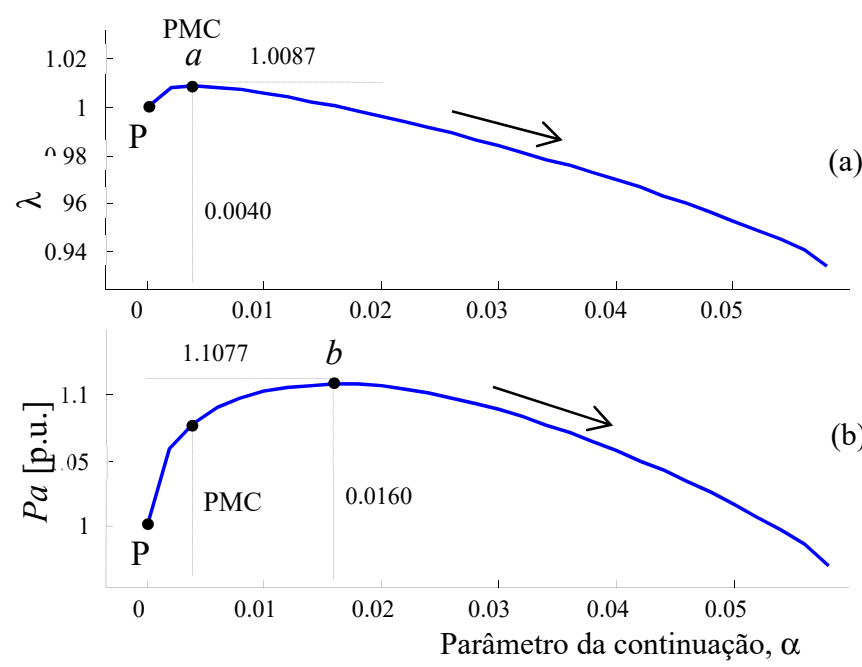

Figura 5. Efeito do parâmetro de continuação $\alpha$ na curva P-V do sistema 638 barras: (a) $\lambda$ como função do parâmetro $\alpha$, (b) $P a$ como função do parâmetro $\alpha$. 
TABELA I

DETERMINANTES NO PONTO “ $a$ ” CONSIDERANDO $\lambda$ COMO PARÂMETRO OU O FCC CONVENCIONAL DO SISTEMA 638 BARRAS

\begin{tabular}{|c|c|c|}
\hline $\begin{array}{c}\text { Fator de } \\
\text { carregamento, } \lambda\end{array}$ & $\begin{array}{c}\left|\mathbf{J}_{\mathbf{m}}\right|, \lambda \text { como parâmetro, } \mathrm{n} \\
=58\end{array}$ & $|\mathbf{J}|, \mathrm{n}=58$ \\
\hline$\ldots \ldots$ & $\ldots \ldots$ & $\ldots \ldots$ \\
\hline 1.00831464813 & $0.2822600 \times 10^{2086}$ & $0.1637132 \times 10^{2088}$ \\
\hline 1.00861029129 & $0.0147500 \times 10^{2086}$ & $0.0085538 \times 10^{2088}$ \\
\hline$* 1.00872723258$ & $* 0.0002600 \times 10^{2086}$ & $* 0.0001489 \times 10^{2088}$ \\
\hline$* 1.00867400107$ & $*-0.0001600 \times 10^{2086}$ & $*-0.0000916 \times 10^{2088}$ \\
\hline 1.00845869807 & $-0.0000600 \times 10^{2086}$ & $-0.0000325 \times 10^{2088}$ \\
\hline 1.00808897885 & $-0.0000200 \times 10^{2086}$ & $-0.0000095 \times 10^{2088}$ \\
\hline$\ldots \ldots$ & $\ldots \ldots$ & $\ldots \ldots$. \\
\hline
\end{tabular}

* PMC (singularidade no ponto " $a$ " quando da parametrização por $\lambda$ ou considerando o FCC convencional)

TABELA II

DETERMINANTES NO PONTO “ $b$ " CONSIDERANDO $P a$ COMO PARÂMETRO DO SISTEMA 638 BARRAS

\begin{tabular}{|c|c|c|}
\hline $\begin{array}{c}\text { Fator de } \\
\text { carregamento, } \lambda\end{array}$ & $\begin{array}{c}\left|\mathbf{J}_{\mathbf{m}}\right|, P a \text { como parâmetro, } \mathrm{n} \\
=58\end{array}$ & Pa \\
\hline$\ldots \ldots \ldots$ & $\ldots \ldots \ldots$ & $\ldots \ldots \ldots$ \\
\hline 1.0052020307 & $0.73935960 \times 10^{2081}$ & 1.10358760683 \\
\hline 1.0041575847 & $0.25057501 \times 10^{2081}$ & 1.10540549636 \\
\hline 1.0029936604 & $0.08926044 \times 10^{2081}$ & 1.10669613384 \\
\hline$* 1.0017138576$ & $* 0.02784377 \times 10^{2081}$ & $* 1.10746006593$ \\
\hline$* 1.0003209911$ & $*-0.00113841 \times 10^{2081}$ & $* 1.10769701624$ \\
\hline 0.9988170175 & $-0.02132781 \times 10^{2081}$ & 1.10740575545 \\
\hline$\ldots \ldots \ldots$ & $\ldots \ldots \ldots$. & $\ldots \ldots \ldots$ \\
\hline
\end{tabular}

* (singularidade no ponto “ $b$ " quando da parametrização por $P a$ )

\section{Resultados para o Sistema Sul Sudeste Brasileiro de 787 barras}

Para o sistema Sul-Sudeste Brasileiro de 787 barras, as matrizes Jacobianas são da ordem $1461 \times 1461$ para o FCC convencional e $1462 \times 1462$ para o FCC parametrizado.

Os valores de n no algoritmo proposto, para que seja possível a visualização e plotagem dos gráficos dos determinantes das matrizes $\mathbf{J}$ e $\mathbf{J}_{\mathbf{m}}$ do sistema de 787 barras, foram entre 35 e 105 , ver tabela 6. Para este sistema, o valor de $n$ adotado foi de 35 , com isso, o valores reais dos determinantes para o FCC parametrizado obtido por meio da equação:

$$
|\mathbf{J}|=\mathrm{n}^{\mathrm{m}} \cdot|\mathbf{A}|=35^{1462} \cdot|\mathbf{A}|
$$

são apresentados nas tabelas 3 e 4 .

A Fig. 6(a) e (b) apresentam as curvas P-V da barra crítica $\left(\mathrm{V}_{576}\right)$ e $\lambda-P a$ e os seus respectivos pontos críticos " $a$ " e " $b$ " do sistema 787 barras. Nota-se que esses pontos estão bem próximos um do outro. Considerando o FCC convencional ou o FCC parametrizado por $\lambda$, a singularidade da matriz $\mathbf{J}$ e $\mathbf{J}_{\mathbf{m}}$ ocorre no ponto " $a$ " (1.1272), conforme apresentado na Fig. 6(a) e (b) e na tabela 3. Caso $P a$ for utilizado como parâmetro, a singularidade de $\mathbf{J}_{\mathbf{m}}$ ocorre no ponto “ $b$ " (1.12669), logo após o ponto " $a$ ", esses resultados podem ser observados na Fig. 6 (a) e (b) e na tabela 4.

Para os demais parâmetros, tensão na barra crítica $\left(\mathrm{V}_{576}\right)$ e parábolas no plano $\lambda-P a$, a singularidade da matriz $\mathbf{J}_{\mathbf{m}}$ é removida possibilitando a obtenção completa da curva $\mathrm{P}-\mathrm{V}$.

Os valores dos determinantes para todos os métodos aqui estudados referentes aos pontos críticos " $a$ " e " $b$ " são apresentados na Fig. 7 (a) e (b). Observa-se que o valor do determinante muda de sinal correspondente aos pontos críticos, comprovando a singularidade da matriz.

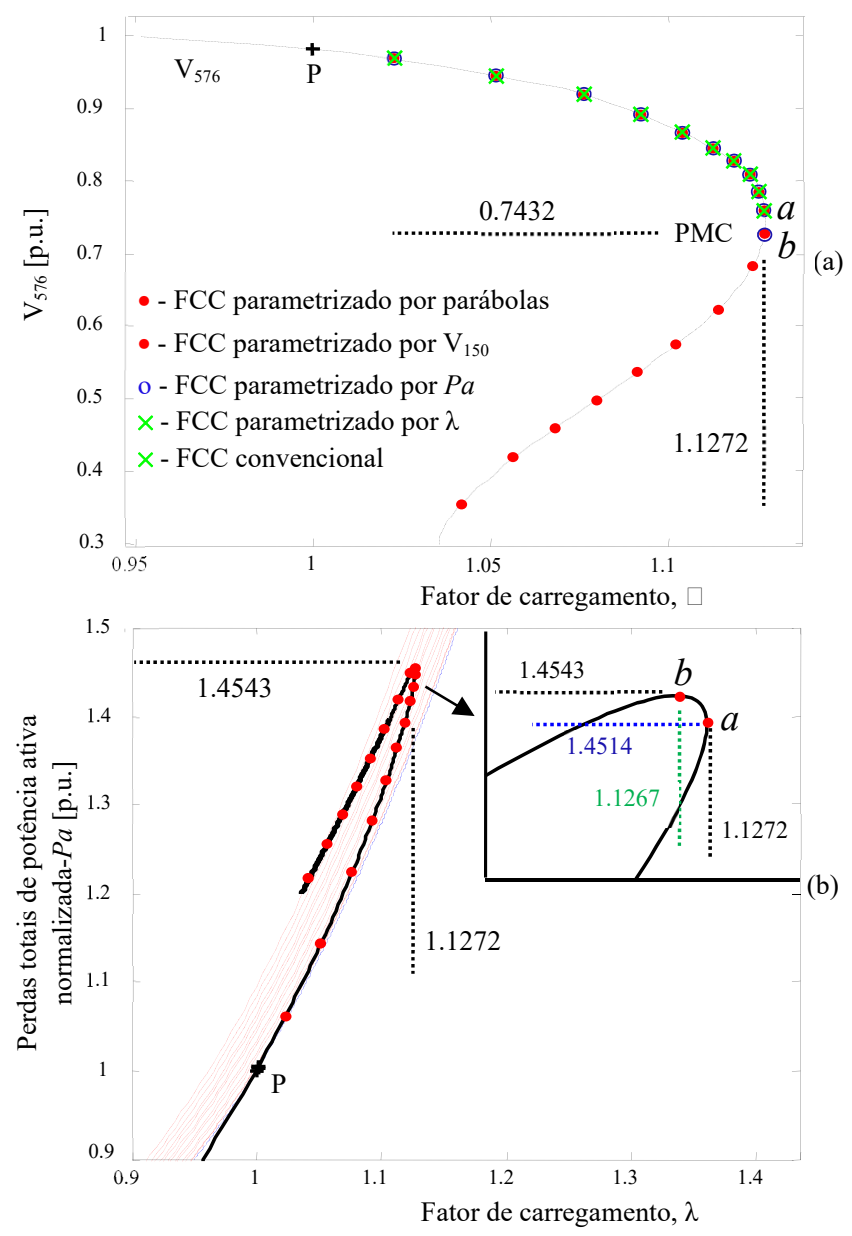

Figura 6. Comparação dos métodos no sistema 787 barras, (a) resultados na curva $\mathrm{P}-\mathrm{V}$, (b) resultados na curva $\lambda-P a$.

TABELA III

DETERMINANTES NO PONTO “ $a$ ” CONSIDERANDO $\lambda$ COMO PARÂMETRO OU O FCC CONVENCIONAL DO SISTEMA 787 BARRAS

\begin{tabular}{|c|c|c|}
\hline $\begin{array}{c}\text { Fator de } \\
\text { carregamento, } \lambda\end{array}$ & $\left|\mathbf{J}_{\mathbf{m}}\right|, \lambda$ como parâmetro, $\mathrm{n}=$ & $|\mathbf{J}|, \mathrm{n}=35$ \\
\hline & & \\
\hline 1.1272627472 & $0.342272515 \times 10^{2251}$ & $0.119795380 \times 10^{2251}$ \\
\hline 1.1272826730 & $0.070367439 \times 10^{2251}$ & $0.024628603 \times 10^{2251}$ \\
\hline * 1.1272853699 & $* 0.009325756 \times 10^{2251}$ & $* 0.003264014 \times 10^{2251}$ \\
\hline * 1.1272709258 & $*_{-}-0.001727464 \times 10^{2251}$ & $*_{-} 0.000604612 \times 10^{2251}$ \\
\hline 1.1272394275 & $-0.002327364 \times 10^{2251}$ & $-0.000814577 \times 10^{2251}$ \\
\hline 1.1271909607 & $-0.001443955 \times 10^{2251}$ & $-0.000505384 \times 10^{2251}$ \\
\hline & & \\
\hline
\end{tabular}

* PMC (singularidade no ponto " $a$ " quando da parametrização por $\lambda$ ou considerando o FCC convencional) 
TABELA IV

DETERMINANTES NO PONTO “ $b$ ” CONSIDERANDO Pa COMO PARÂMETRO DO SISTEMA 787 BARRAS

\begin{tabular}{|c|c|c|}
\hline $\begin{array}{c}\text { Fator de } \\
\text { carregamento, } \lambda\end{array}$ & $\begin{array}{c}\left|\mathbf{J}_{\mathbf{m}}\right|, P a \text { como parâmetro, } \mathrm{n} \\
=35\end{array}$ & $\mathrm{~Pa}$ \\
\hline$\ldots \ldots \ldots$ & $\ldots \ldots \ldots$ & $\ldots \ldots \ldots$ \\
\hline 1.1270434606 & $0.894728134 \times 10^{2249}$ & 1.4540806840 \\
\hline 1.1269445947 & $0.245166390 \times 10^{2249}$ & 1.4542373030 \\
\hline 1.1268290957 & $0.057388499 \times 10^{2249}$ & 1.4543151026 \\
\hline$* 1.1266970458$ & $* 0.007505177 \times 10^{2249}$ & $* 1.4543147346$ \\
\hline$* 1.1265485274$ & $*-0.003197771 \times 10^{2249}$ & $* 1.4542368934$ \\
\hline 1.1263836224 & $-0.003907523 \times 10^{2249}$ & 1.4540823145 \\
\hline$\ldots \ldots \ldots$ & $\ldots \ldots \ldots$ & $\ldots \ldots \ldots$ \\
\hline
\end{tabular}

* (singularidade no ponto " $b$ ” quando da parametrização por $P a$ )
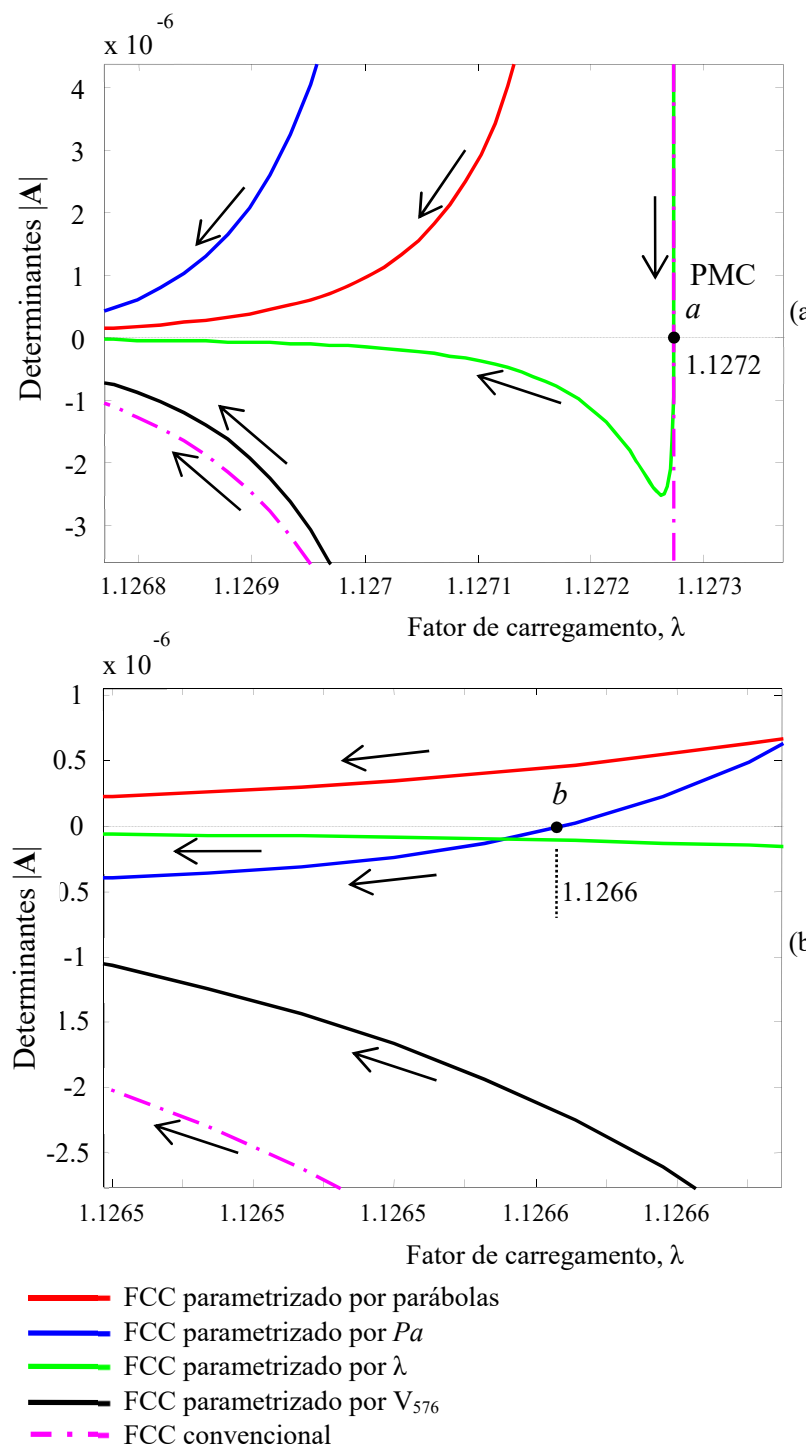

(b)

Figura 7. Determinantes dos métodos para o sistema 787 barras: (a) performance dos métodos no ponto " $a$ ", (b) performance dos métodos no ponto " $b$ ".

Resultados para o Sistema de 904 barras Localizado no Sudoeste Americano
Para o sistema de 904 barras, as matrizes Jacobianas são da ordem $1461 \times 1461$ para o FCC convencional e $1676 \times 1676$ para o FCC parametrizado.

Os valores de $n$ na tabela 6 foram entre 25 e 45 . Para este sistema, o valor de $\mathrm{n}$ adotado foi de 35 , com isso, o valores reais dos determinantes para o FCC parametrizado obtido por meio da equação:

$$
|\mathbf{J}|=\mathrm{n}^{\mathrm{m}} \cdot|\mathbf{A}|=35^{1676} \cdot|\mathbf{A}|
$$

são apresentados nas tabelas 5.

A Fig. 8(a) e (b) apresentam as curvas P-V da barra crítica $\left(\mathrm{V}_{138}\right)$ e $\lambda-P a$ e os seus respectivos pontos críticos " $a$ " e " $b$ " do sistema 904 barras. Nota-se que esses pontos são praticamente os mesmos. Considerando o FCC convencional ou o FCC parametrizado por $\lambda$, a singularidade da matriz $\mathbf{J}$ e $\mathbf{J}_{\mathbf{m}}$ ocorre no ponto " $a$ " (1.1991), conforme apresentado na Fig. 8(a) e (b) e na tabela 5. Caso $P a$ for utilizado como parâmetro, a singularidade de $\mathbf{J}_{\mathbf{m}}$ ocorre no ponto " $b$ " (1.1991), que corresponde praticamente ao ponto " $a$ " (mesmo ponto). Para este sistema, utilizando as parábolas como parâmetro, a singularidade de $\mathbf{J}_{\mathbf{m}}$ também ocorre no ponto " $b$ ".

Já para a tensão da barra crítica $\left(\mathrm{V}_{138}\right)$ como parâmetro, a singularidade da matriz $\mathbf{J}_{\mathbf{m}}$ é removida possibilitando a obtenção completa da curva P-V. Os valores do determinante de $\mathbf{J}_{\mathbf{m}}$ parametrizado por $\mathrm{V}_{138}$ podem ser vistos na última coluna da tabela 5 .

Os valores dos determinantes para todos os métodos aqui estudados referentes aos pontos críticos " $a$ " e " $b$ " são apresentados na Fig. 9 e tabela 5. Observa-se que o valor do determinante muda de sinal correspondente aos pontos críticos, comprovando a singularidade da matriz $\mathbf{J}_{\mathbf{m}}$ parametrizado por $\lambda, P a$ e parábolas e singularidade da matriz $\mathbf{J}$ para o FCC convencional, ver tabela 5 .

A Fig. 9 apresenta os resultados dos determinantes da tabela 5 geometricamente. Observa-se que todas as curvas intersectam o eixo das abscissas no ponto " $a$ " (singularidade) com exceção $\mathrm{o}$ método parametrizado por $\mathrm{V}_{138}$ que não intersecta o eixo $\mathrm{x}$ comprovando a não singularidade da matriz $\mathbf{J}_{\mathbf{m}}$. 

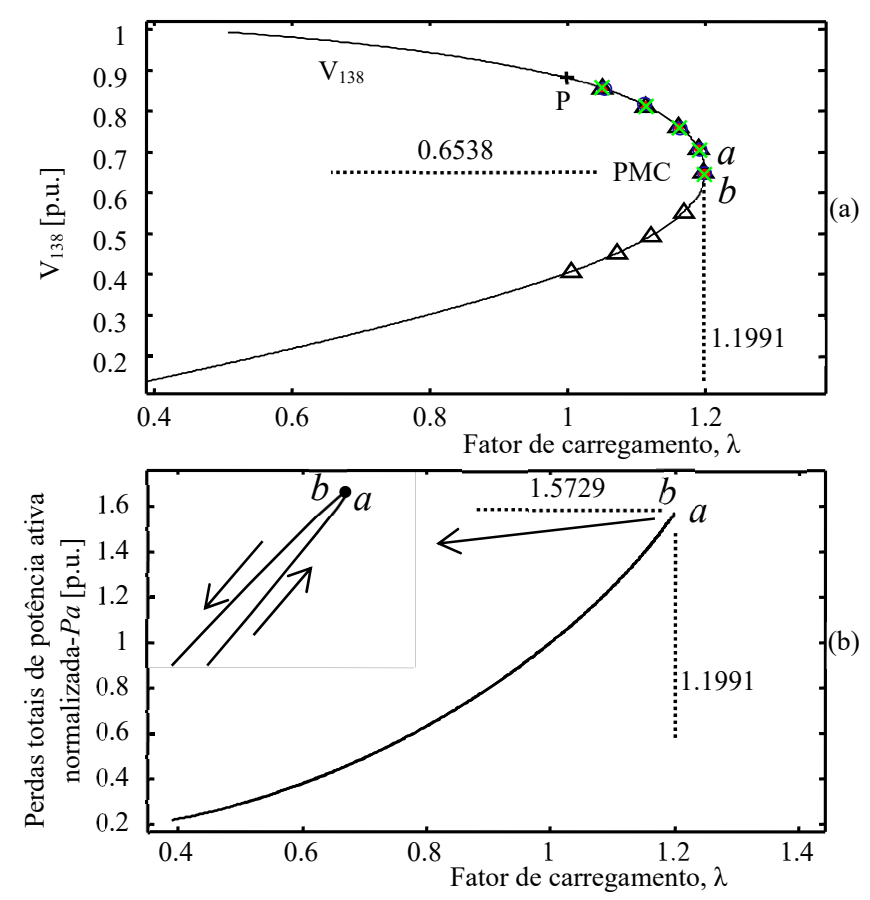

- FCC parametrizado por parábolas

$\Delta$ - FCC parametrizado por $\mathrm{V}_{138}$

o - FCC parametrizado por $\mathrm{Pa}$

$\times$ - FCC parametrizado por $\square$

$x$ - FCC convencional

Figura 8. Comparação dos métodos no sistema 904 barras, (a) resultados na curva $\mathrm{P}-\mathrm{V}$, (b) resultados na curva $\lambda-P a$.

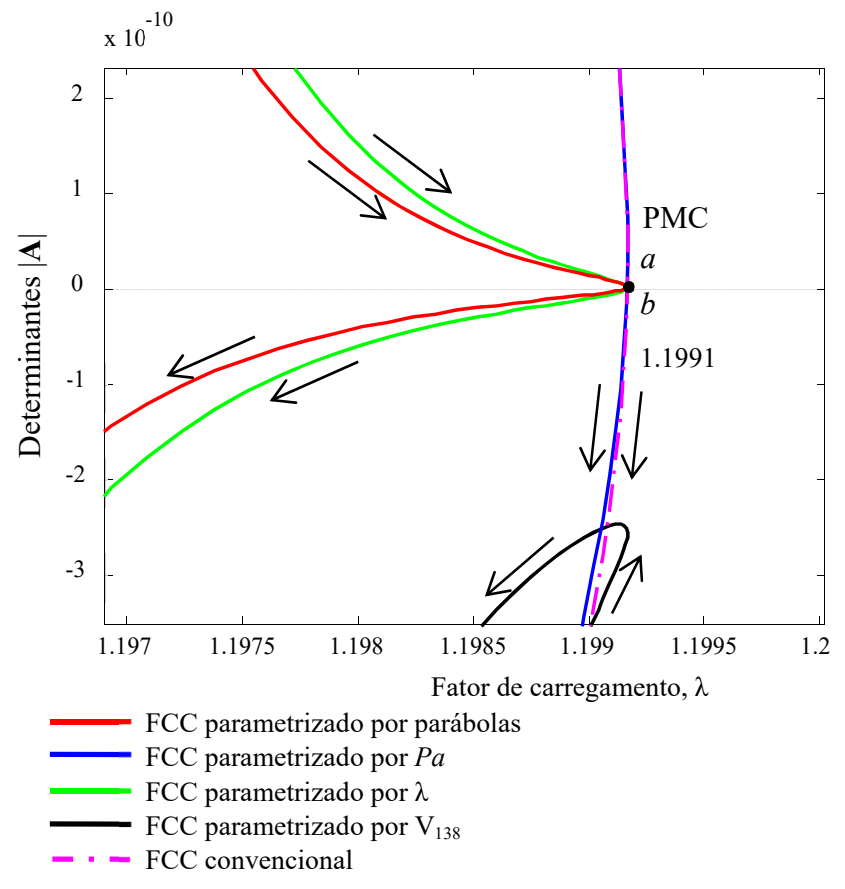

Figura 9. Determinantes dos métodos para o sistema 904 barras: (a) performance dos métodos no ponto " $a$ " $\mathrm{e}$ "b".
TABELA V

DETERMINANTES NO PONTO “ $a$ ” $E$ “ $b$ " CONSIDERANDO VÁRIOS PARÂMETROS DO SISTEMA 904 BARRAS

\begin{tabular}{|c|c|c|c|c|c|}
\hline $\begin{array}{c}\text { Fator de } \\
\text { carregamento, } \\
\lambda\end{array}$ & $\begin{array}{c}\left|\mathbf{J}_{\mathbf{m}}\right|, \lambda \\
\text { como } \\
\text { parâmetro, } \\
\mathrm{n}=35 \\
\mathrm{x} 10^{2576}\end{array}$ & $\begin{array}{c}\quad|\mathbf{J}| \\
\mathrm{n}=35 \\
\mathrm{x} 10^{2578}\end{array}$ & $\begin{array}{c}\left|\mathbf{J}_{\mathbf{m}}\right| \\
P a \text { como } \\
\text { parâmetro, } \\
\mathrm{n}=35 \\
\mathrm{x} 10^{2578}\end{array}$ & $\begin{array}{c}\left|\mathbf{J}_{\mathbf{m}}\right| \\
\text { Parábolas } \\
\text { como } \\
\text { parâmetro, } \\
\mathrm{n}=35 \\
\text { x } 10^{2576}\end{array}$ & $\begin{array}{c}\left|\mathbf{J}_{\mathbf{m}}\right| \\
\mathrm{V}_{138} \text { como } \\
\text { parâmetro, } \\
\mathrm{n}=35 \\
\mathrm{x} 10^{2578}\end{array}$ \\
\hline & & & & & \\
\hline 1.199160 & 0.34454 & 0.12059 & 0.12579 & 0.28192 & -0.26807 \\
\hline 1.199167 & 0.18893 & 0.06612 & 0.07555 & 0.17041 & -0.26056 \\
\hline$* 1.199168$ & $* 0.04203$ & $* 0.01471$ & $* 0.02818$ & $* 0.06411$ & $* *-0.25470$ \\
\hline *1.199164 & $*_{-0.09891}$ & $*_{-0.0346}$ & $*_{-0}-01719$ & *-0.03886 & $* *_{-} 0.25040$ \\
\hline 1.199154 & -0.23643 & -0.08275 & -0.06138 & -0.14029 & -0.24756 \\
\hline 1.199139 & -0.37295 & -0.13053 & -0.10517 & -0.19329 & -0.24616 \\
\hline & & & & & \\
\hline
\end{tabular}

* PMC (singularidade no ponto " $a$ " e " $b$ " quando da parametrização por $\lambda$ ou considerando o FCC convencional, $P a$ e parábolas), ${ }^{* *}$ sem singularidade.

A tabela 6 a seguir apresenta os valores do intervalo de $n$ para que seja possível visualizar e plotar os gráficos dos determinantes de cada sistema de grande porte por meio do software MATLAB.

TABELA VI

INTERVALO DOS VALORES DE $\mathrm{n}$ DO ALGORITMO PROPOSTO PARA CADA SISTEMA

\begin{tabular}{|c|c|}
\hline \multicolumn{2}{|c|}{ SISTEMA } \\
\hline Sistemas & $\begin{array}{c}\text { Valores de } \mathrm{n} \text { para } \\
\text { obtenção dos } \\
\text { determinantes }\end{array}$ \\
\hline 638 barras & $35 \leq \mathrm{n} \leq 105$ \\
\hline 787 barras & $25 \leq \mathrm{n} \leq 59$ \\
\hline 904 barras & $25 \leq \mathrm{n} \leq 45$ \\
\hline
\end{tabular}

\section{CONCLUSÃO}

Nesta parte foram apresentados os resultados referentes as metodologias apresentadas na primeira parte do trabalho. Uma alternativa para viabilizar a representação gráfica dos valores do determinante em função do carregamento $\lambda$ foi apresentada, que é a redução dos seus valores através da divisão dos elementos da matriz por $\mathrm{n}$. Com base nesta representação gráfica, neste trabalho também se apresentou a análise de algumas das várias propostas utilizadas para a melhoria do desempenho do fluxo de carga continuado visando à obtenção do ponto de máximo carregamento de sistemas elétricos de potência sem os problemas numéricos relacionados à singularidade da matriz $\mathbf{J}$.

\section{AGRADECIMENTOS}

Os autores agradecem a UNESP e ao CNPq pelo apoio financeiro.

\section{REFERÊNCIAS}

[1] A. Bonini Neto, D. A. Alves. Singularities Analysis of the Jacobian Matrix Modified in the Continuation Power Flow: Mathematical Modeling. Revista IEEE América Latina, v. 14, p. 4750-4756, 2016. 
[2] V. Ajjarapu and C.Christy. The Continuation Power Flow: a Tool for Steady State Voltage Stability Analysis. IEEE Trans. on Power Systems, vol. 7, n. 1, February, pp. 416-423, 1992.

[3] E Garbelini, D. A. Alves, A. Bonini Neto, E. Righeto, L. C. P. da Silva, C. A. Castro. An efficient geometric parameterization technique for the continuation power flow. Electric Power Systems Research, vol. 77, pp. 71-82, 2007.

[4] MATHWORKS. Matlab (MATrix LABoratory). Disponível em: $<$ http://www.mathworks.com>. Acesso em 21 de maio de 2016.

[5] E. M. Magalhães, A. Bonini Neto, D. A. Alves. Técnica de Parametrização Geométrica para o Fluxo de Carga Continuado Baseado nas Variáveis Fator de Carregamento e Perda Total de Potência Ativa. In: Décimo Quinto Encontro Regional Ibero-Americano do Cigré - XV ERIAC, Foz do Iguaçu, p. 1-8, 2013.

[6] A. Bonini Neto, D. A. Alves. Técnica de Parametrização Geométrica para o Fluxo de Carga Continuado Baseado nas Variáveis Tensão Nodal e Fator de Carregamento. Controle \& Automação (Impresso), v. 19, p. 350366, 2008

[7] A. Bonini Neto, E. M. Magalhães, D. A. Alves. Dishonest Newton Method Applied in Continuation Power Flow Through a Geometric Parameterization Technique. Revista IEEE América Latina, v. 14, p. 161$170,2016$.

[8] E. M. Magalhães, A. Bonini Neto, D. A. Alves. A parameterization technique for the continuation power flow developed from the analysis of power flow curves. Mathematical Problems in Engineering, vol. 2012, p. $1-24,2012$.

[9] A. Bonini Neto, D. A. Alves. Improved geometric parameterization techniques for continuation power flow. IET Generation, Transmission \& Distribution; vol. 4, p. 1349-1359. 2010.

[10] A. Bonini Neto, E. M. Magalhães, D. A. Alves. Obtaining the Maximum Loading Point of Electric Power Systems using the Decoupled Methods of Newton. Revista IEEE América Latina, v. 14, p. 371-379, 2016.

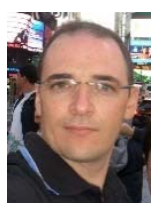

A. Bonini Neto, graduado em Matemática pela Faculdade de Dracena - UNIFADRA, Brasil, em 2002, mestrado e doutorado em Engenharia Elétrica pela Universidade Estadual Paulista UNESP, campus de Ilha Solteira em 2006 e 2011 respectivamente. Trabalha com pesquisas na área de matemática aplicada na engenharia, técnicas de parametrização no fluxo de carga continuado. Atualmente é professor assistente doutor da Universidade Estadual Paulista - UNESP, campus de Tupã.

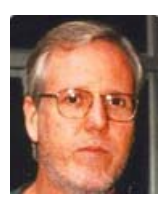

D. A. Alves, graduado em Engenharia Elétrica pela Universidade de São Paulo - USP, Brasil em 1980, mestrado e doutorado em Engenharia Elétrica pela Universidade Campinas - UNICAMP em 1988 e 2000 respectivamente. Trabalha com pesquisas na área de fluxo de carga continuado. Atualmente é professor adjunto da Universidade Estadual Paulista - UNESP, campus de Ilha Solteira. 